\title{
Pulmonary oligometastases treated by stereotactic body radiation therapy (SBRT): a single institution's experience
}

\author{
Guangjin Chai", Yutian Yin", Xiaoying Zhou, Qilong Hu, Bo Lv, Zhaohui Li, Mei Shi, Lina Zhao \\ Department of Radiation Oncology, Xijing Hospital, Air Force Medical University, Xi'an, China \\ Contributions: (I) Conception and design: L Zhao, M Shi; (II) Administrative support: None; (III) Provision of study materials or patients: None; \\ (IV) Collection and assembly of data: G Chai, X Zhou, Q Hu, B Lv, Z Li; (V) Data analysis and interpretation: Y Yin; (VI) Manuscript writing: All \\ authors; (VII) Final approval of manuscript: All authors. \\ "These authors contributed equally to this work. \\ Correspondence to: Lina Zhao; Mei Shi. Department of Radiation Oncology, Xijing Hospital, Air Force Medical University, 15 West Changle Road, \\ Xi’an, China. Email: zhaolinazln@outlook.com; mshi82@hotmail.com.
}

Background: To investigate the effect of stereotactic body radiation therapy (SBRT) on pulmonary oligometastases and to analyze the clinical factors and dose parameters affecting local recurrence-free survival (LRFS) and overall survival (OS).

Methods: This study retrospectively enrolled a total of 84 patients (148 lesions) treated in our department from May 2015 to November 2018. Pulmonary oligometastases was defined as up to 5 metastatic lesions in the lung and with both the primary tumor and any extra-thoracic metastases being controlled. Patients receiving a $\mathrm{BED}_{10}$ (biological effective dose, $\alpha / \beta=10$ ) of $\mathrm{SBRT} \geq 75$ Gy and a dose/fraction $\geq 4$ Gy were enrolled. The patient group consisted of 52 men (61.9\%) and 32 women (38.1\%), with a median age 56 years (range, 29-80 years). Median tumor diameter was $1.71 \mathrm{~cm}$ (range, 1.2-5.0 cm). The BED ${ }_{10}$ was $75-119$ Gy in 4-15 fractions. Univariate and multivariate Cox regression analyses were performed on factors predicting the outcomes.

Results: All patients completed the treatment as planned, and the median follow-up time was 20.3 months. The median OS for the entire group was 34.3 months, with an actuarial 1-, 2-, 3- and 5-year OS of 74.7\%, $59.4 \%, 49.7 \%$, and 36.8\%, respectively. Among the 148 lesions in the whole group, 19 (12.8\%) lesions had local recurrence (LR). The median LRFS time for all patients was 56.9 months. The LRFS rate was $93.6 \%$, $83.5 \%, 81.4 \%$, and $76.6 \%$ at 1, 2, 3, and 5 years, respectively. No patient developed acute grade 3 or 4 toxicity. On univariate analysis, age $\geq 63$ years old, primary site of colorectal cancer, $\mathrm{BED}_{10}<85.2$ Gy, pathological type of adenocarcinoma, planning target volume (PTV) $\min \mathrm{BED}_{10}<76.6 \mathrm{~Gy}$, and gross tumor volume (GTV) $\geq 8.8 \mathrm{cc}$, were significantly associated with poorer LRFS. Multivariate analysis showed that age $\geq 63$ years old, primary site of colorectal cancer, and PTV min $\mathrm{BED}_{10}<76.6$ Gy were significant risk factors affecting LRFS. Conclusions: SBRT is feasible for pulmonary oligometastasis with favorable local control and minimal toxicity. Multiple dose parameters, instead of a prescription dose only, in combination with clinical parameters, should be considered for optimal local control.

Keywords: Stereotactic body radiation therapy (SBRT); pulmonary oligometastases; local recurrence-free survival (LRFS); overall survival (OS)

Submitted Jun 29, 2020. Accepted for publication Aug 05, 2020.

doi: $10.21037 /$ tlcr-20-867

View this article at: http://dx.doi.org/10.21037/tlcr-20-867 


\section{Introduction}

Oligometastasis, as described by Hellman and Weichselbaum, is the state preceding the extensive distribution of cancer cells (1). Under this paradigm, patients with oligometastases should be offered additional curative treatment instead of the systemic treatment alone. A recent phase II study demonstrated that local consolidative therapy can improve progression-free survival (PFS) and overall survival (OS) compared to maintenance therapy/observation in oligometastatic non-small cell lung cancer (NSCLC) $(2,3)$. Stereotactic body radiation therapy (SBRT), also known as stereotactic ablative radiotherapy (SABR), is a modern radiation technique characterized by delivering a conformal high radiation dose in a few fractions while sparing the surrounding healthy tissues (4). SBRT has been used to treat inoperable early NSCLC with high local control rate and low toxicities (5,6). A randomized study also indicates that SBRT is as effective as surgery in patients who are fit to undergo surgery (7). Given these encouraging results for primary early stage NSCLC, there is increasing interest in using SBRT as a local treatment modality for patients with pulmonary oligometastases. The randomized phase 2 SABR-COMET trial showed SBRT was able to improve OS in patients with oligometastatic lesions (8).

The lung is one of the most common metastatic sites of malignant tumors. Existing trials of SBRT for lung oligometastases report 2-year local control rates of approximately $80 \%$, 2-3-year disease-free survival (DFS) rates of approximately $20 \%$, and the 2-3-year OS rates of $25-40 \%$, which are comparable to surgical series (9-12). Furthermore, several studies have reported some prognostic factors of pulmonary oligometastases treated with SBRT $(10,13)$. It has been widely accepted that at least a prescribed biologically effective dose (BED) greater than $100 \mathrm{~Gy}$ (assuming an $\alpha / \beta$ ratio of 10 ) is needed to ensure good local control for early stage lung cancer $(6,7,14-16)$. A few studies also found that a higher BED was needed for better local control in lung oligometastases $(17,18)$. However, the optimal dose for oligometastatic lung tumors has not yet been determined. Other dose parameters should also be considered besides the prescribed dose. Our previous study indicated that even if the prescribed dose is the same, the dose received by the planning target volume (PTV) can be quite different while the PTV D95 and mean dose should be considered for optimal local control for early stage early stage NSCLC receiving SBRT (19). To our knowledge, no study has thus far investigated the detailed optimal dose parameters for pulmonary oligometastases treated by SBRT, from a radiation oncology perspective. Therefore, the purpose of the present research was to analyze the clinical factors and dose parameters affecting local control in patients receiving a $\mathrm{BED}_{10}$ of SBRT $\geq 75$ Gy and a dose/ fraction $\geq 4$ Gy with a controlled primary tumor and up to five lung metastatic lesions (20).

We present the following article in accordance with the STROBE reporting checklist (available at http://dx.doi. org/10.21037/tlcr-20-867).

\section{Methods}

\section{Patients}

A total of 84 patients with pulmonary oligometastases (148 lesions) were treated with image-guided SBRT in our department from May 2015 to November 2018. The inclusion criteria were the following: (I) oligometastatic disease (up to 5 metastatic lesions in the lung and 3 metastatic organs); (II) lung metastases $\leq 5 \mathrm{~cm}$ in size; (III) control of both the primary tumor and any extra-thoracic metastases; (IV) an expected minimum life expectancy of 6 months; (V) good performance status (PS) (0 or 1), and (VI) $\mathrm{BED}_{10} \geq 75 \mathrm{~Gy}$ and dose/fraction $\geq 4$ Gy. If patients received prior thoracic irradiation, it was required that the current irradiation volume was implemented outside the previous irradiated field, and was set to a 20-Gy or higher isodose line. The analysis is in compliance with the principles of the Declaration of Helsinki (as revised in 2013). This study was approved by the institutional review board of Xijing Hospital (no. KY20202002-F-1). Written informed consent was obtained from each of the participants. The diagnostic imaging consisted of at least a computed tomography (CT) scan of the thorax/abdomen or positron emission tomography (PET)/CT which was not older than 1 month at the time of SBRT referral.

\section{SBRT protocol}

Four-dimensional (4D) CT simulation was used in all the patients to account for the respiratory motion. The gross tumor volume (GTV) was drawn as a visible tumor using CT lung window imaging. The internal target volume (ITV) was composed of the combinations of the GTV contours on 10 4D phases of respiration. No expansion from ITV to the clinical target volume (CTV) was used. The PTV was the CTV plus a $5 \mathrm{~mm}$ three-dimensional 
(3D) expansion. A dose of 48-70 Gy in 6-15 fractions was prescribed to the PTV. The dose-fractionation schemes were prescribed by the radiation oncologists based on the tumor volume, location, and dose constraints of normal tissues. Furthermore, 6-12 coplanar or non-coplanar 6-MV photon beams, or 1-3 arcs were used to optimize the SBRT plans using intensity-modulated radiation therapy (IMRT) or volumetric-modulated arc therapy (VMAT). Daily online cone-beam CT (CBCT) was performed to verify the tumor location. Treatment was delivered on consecutive weekdays.

\section{Follow-up}

Patients were followed every 3 months for the first 2 years after treatment and every 6 months thereafter. Clinical visits and CT scans were performed at each follow-up. PET/CT scans were obtained when suspicious abnormalities were present CT imaging. Local recurrence (LR) was defined as CT evidence of progressive soft tissue abnormalities in the irradiated area, corresponding to PET-avid areas [maximum standardized uptake value (SUVmax) >5] more than 6 months after SBRT (21) or positive biopsy findings. Toxicities were graded using the Common Terminology Criteria of Adverse Events Version 4.0 (CTCAE v.4.0).

\section{Endpoint}

Local recurrence-free survival (LRFS) was calculated from the date of the beginning of SBRT to the date of first LR or last contact date. For the patients who died before the onset of LR, we calculated LRFS from the date of the beginning of SBRT to the date of death. OS was defined as the length of time from the beginning of SBRT to death by any cause.

\section{Statistical analysis}

All dose information for this study, including prescribed $\mathrm{BED}_{10}, \mathrm{BED}_{10}$ of PTV max dose (maximum PTV dose), $\mathrm{BED}_{10}$ of PTV mean dose (mean PTV dose), BED ${ }_{10}$ of PTV min dose (minimum PTV dose), BED ${ }_{10}$ of PTV D95 (minimum dose to $95 \%$ of PTV volume), and $\mathrm{BED}_{10}$ of PTV D99 (minimum dose to $99 \%$ of PTV volume), were calculated using the following linear-quadratic model: $\mathrm{BED}_{10}=$ number of fractions dose per fraction $(1+$ dose per fraction/10). The continuous variables were divided into 2 subgroups at their cutoff values identified by local control status through receiver operating characteristic (ROC) curves. Kaplan-Meier curves in the survival analysis were used to present the percent survival of 1-, 2-, 3-, and 5 -year LRFS and OS; every two subgroups were compared by of log-rank tests. To analyze the influence of potential risk factors on LRFS and OS, we used univariable and multivariable Cox analyses. The factors with statistical significance after screening by univariable cox regression analysis ( $\mathrm{P}$ value: entry 0.05 , removal 0.10 ), were put into the multivariable Cox regression model. Forest maps could more intuitively show the risk factors and protective factors for LRFS and OS, along with their hazard ratios (HRs) and $95 \%$ confidence interval (CI). The nomograms were based on the results of multivariate Cox regression analysis and used to predict the combined effect of the influencing factors on LRFS and OS. We used Epidata 3.1 to establish the database. SPSS 24.0 (IBM) statistical software and $\mathrm{R}$ 3.6.1 were used for data analysis. A two-tailed $\mathrm{P}$ value $<0.05$ was considered to be statistically significant.

\section{Results}

\section{Patient characteristics}

All patients completed the treatment as planned, and the characteristics of patients and lung tumors are shown in Table 1. A total of 84 patients (148 lesions) were treated with SBRT, including 52 males and 32 females, with a median age of 58.5 years old. The primary tumors of the 148 pulmonary metastatic lesions were as follows: 42 from head and neck cancer, 39 from esophageal cancer, 27 from colorectal cancer (CRC), 9 from cervical cancer, 9 from breast cancer, and 22 from other sites. For disease type, 55 were squamous cell carcinoma, 47 were adenocarcinoma, and 46 were other histologic types. Furthermore, $41.7 \%$ patients $(35 / 84)$ had metastatic lesions in more than one lung, and all lesions were irradiated. Additionally, $14.3 \%$ patients $(12 / 84)$ had extra thoracic metastases with both the primary tumor and any extra-thoracic metastases being well controlled. For treatment dosage, 49 lesions (33.1\%) were treated with $60 \mathrm{~Gy}$ in 15 fractions, 48 lesions (32.4\%) received $60 \mathrm{~Gy}$ in 10 fractions, and 14 lesions (9.5\%) received 70 Gy in 10 fractions. Treatment fractions were between 4 and 15 fractions, and the $\mathrm{BED}_{10}$ was $75-119 \mathrm{~Gy}$.

\section{Survival analysis}

The median follow-up time was 20.3 months (range, 4.2-68.1 months), with 47 patients (55.9\%) alive at last follow-up. The median OS time was 34.3 months. The 
Table 1 The characteristics of patients and tumors

\begin{tabular}{|c|c|}
\hline Variables & $M\left(Q_{L}, Q_{U}\right) / n(\%)$ \\
\hline \multicolumn{2}{|l|}{ No. } \\
\hline Patients & 84 \\
\hline Tumors & 148 \\
\hline Age (years) & $56(50,64)$ \\
\hline Tumor diameter $(\mathrm{cm})$ & $1.71(1.12,2.30)$ \\
\hline OTT of SBRT (days) & $15(13,22)$ \\
\hline Fractions & $10(10,15)$ \\
\hline Prescription $\mathrm{BED}_{10}(\mathrm{~Gy})$ & $96.0(84.0,96.0)$ \\
\hline $\mathrm{BED}_{10}$ of PTV mean dose (Gy) & $91.9(88.1,104.5)$ \\
\hline BED $_{10}$ of PTV D95 (Gy) & $85.7(82.1,96.3)$ \\
\hline $\mathrm{BED}_{10}$ of PTV max dose (Gy) & $103.4(94.9,114.8)$ \\
\hline $\mathrm{BED}_{10}$ of PTV min dose (Gy) & $75.6(67.5,85.8)$ \\
\hline BED $_{10}$ of PTV D99 (Gy) & $82.6(78.1,93.1)$ \\
\hline GTV volume $\left(\mathrm{cm}^{3}\right)$ & $6.0(2.9,16.1)$ \\
\hline \multicolumn{2}{|l|}{ Sex } \\
\hline Male & $52(61.9)$ \\
\hline Female & $32(38.1)$ \\
\hline \multicolumn{2}{|l|}{ Primary site } \\
\hline Head and neck & $42(28.4)$ \\
\hline Esophageal & $39(26.3)$ \\
\hline Colorectal & $27(18.2)$ \\
\hline Cervical & $9(6.1)$ \\
\hline Breast & $9(6.1)$ \\
\hline Others & $22(14.9)$ \\
\hline \multicolumn{2}{|l|}{ Dose/fraction } \\
\hline $60 \mathrm{~Gy} / 15 \mathrm{f}$ & $49(33.1)$ \\
\hline $60 \mathrm{~Gy} / 10 \mathrm{f}$ & $48(32.4)$ \\
\hline $70 \mathrm{~Gy} / 10 \mathrm{f}$ & $14(9.5)$ \\
\hline Others & $37(25.0)$ \\
\hline \multicolumn{2}{|l|}{ Histology } \\
\hline Adenocarcinoma & $47(31.8)$ \\
\hline Squamous cell & $55(37.2)$ \\
\hline Other & $46(31.1)$ \\
\hline \multicolumn{2}{|c|}{ Chemotherapy prior to lung SBRT } \\
\hline No & $46(54.8)$ \\
\hline Yes & $38(45.2)$ \\
\hline
\end{tabular}

Table 1 (continued)
Table 1 (continued)

\begin{tabular}{lc}
\hline Variables & $M\left(Q_{\llcorner}, Q_{U}\right) / n(\%)$ \\
\hline Lung metastasis & $49(58.3)$ \\
1 & $29(34.5)$ \\
$2-3$ & $6(7.2)$ \\
$4-5$ & \\
Extra thoracic metastasis & $72(85.7)$ \\
No & $12(14.3)$ \\
Yes & \\
\hline
\end{tabular}

The data was shown as median (lower quartile, upper quartile) or number (percent). OTT, overall treatment time; SBRT, stereotactic body radiation therapy; BED, biological effective dose; PTV, planning target volume; GTV, gross tumor volume.

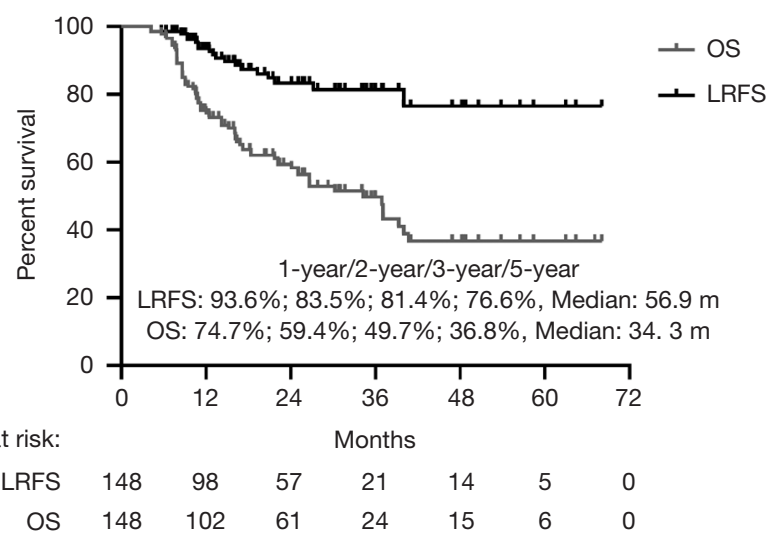

Figure 1 Kaplan-Meier analysis of local recurrence-free survival (LRFS) and overall survival (OS). The median LRFS and OS time and the percent survival of 1-, 2-, 3-, and 5-year of LRFS and OS rate and the number at risk was also presented.

OS at $1,2,3$, and 5 years was $74.7 \%, 59.4 \%, 49.7 \%$, and $36.8 \%$, respectively (Figure 1). In this study, the dominant failure pattern was distant metastasis $(54.7 \%, 46 / 84)$, which occurred in the lung ( 9 patients), bone (14 patients), liver (13 patients), brain (7 patients), and other locations (3 patients).

Fourteen predictive factors were included in the univariable Cox analysis of OS (Table 2), with age, sex, dose/fraction, and histology showing significant difference (Figure S1).

In multivariate analysis (Table 3$)$, age $\geq 63$ years $(\mathrm{P}=0.020$, $\mathrm{HR}=1.793)$, adenocarcinoma $(\mathrm{P}=0.024, \mathrm{HR}=2.354)$ and squamous cell carcinoma $(\mathrm{P}<0.001, \mathrm{HR}=5.677)$ were poor 
Table 2 Univariable Cox analysis of LRFS and OS

\begin{tabular}{|c|c|c|c|c|}
\hline Variables & \multicolumn{2}{|l|}{ LRFS } & \multicolumn{2}{|l|}{ OS } \\
\hline \multicolumn{5}{|l|}{ Age (years) } \\
\hline$<63$ & 1.000 & & 1.000 & \\
\hline$\geq 63$ & $5.531(2.094-14.612)$ & 0.001 & $1.703(1.057-2.746)$ & 0.029 \\
\hline Male & 1.000 & & 1.000 & \\
\hline Female & $0.675(0.256-1.779)$ & 0.427 & $0.387(0.216-0.696)$ & 0.002 \\
\hline \multicolumn{5}{|l|}{ Tumor diameter $(\mathrm{cm})$} \\
\hline$<1.35$ & 1.000 & & 1.000 & \\
\hline$<19$ & 1.000 & & 1.000 & \\
\hline$\geq 19$ & $2.082(0.837-5.180)$ & 0.115 & $0.757(0.448-1.278)$ & 0.297 \\
\hline \multicolumn{5}{|l|}{ Dose/fraction } \\
\hline 60 Gy/15 f & 1.000 & & 1.000 & \\
\hline 60 Gy/10 f & $0.638(0.231-1.764)$ & 0.386 & $1.180(0.612-2.276)$ & 0.621 \\
\hline 70 Gy/10 f & - & 0.978 & $1.164(0.458-2.963)$ & 0.749 \\
\hline Others & $0.354(0.094-1.331)$ & 0.124 & $2.018(1.091-3.733)$ & 0.025 \\
\hline \multicolumn{5}{|l|}{ Primary site } \\
\hline \multicolumn{5}{|l|}{ Histology } \\
\hline Adenocarcinoma & $3.200(0.984-10.412)$ & 0.053 & $2.371(1.135-4.954)$ & 0.022 \\
\hline Squamous & $2.636(0.726-9.579)$ & 0.141 & $6.587(3.263-13.294)$ & $<0.001$ \\
\hline \multicolumn{5}{|l|}{$\mathrm{BED}_{10}(\mathrm{~Gy})$} \\
\hline$<85.2$ & 1.000 & & 1.000 & \\
\hline$\geq 85.2$ & $0.375(0.147-0.954)$ & 0.040 & $1.438(0.867-2.386)$ & 0.159 \\
\hline \multicolumn{5}{|c|}{$\mathrm{BED}_{10}$ of PTV max dose (Gy) } \\
\hline$<97.0$ & 1.000 & & 1.000 & \\
\hline$\geq 97.0$ & $0.450(0.177-1.146)$ & 0.094 & $1.106(0.674-1.816)$ & 0.690 \\
\hline \multicolumn{5}{|c|}{$\mathrm{BED}_{10}$ of PTV mean dose (Gy) } \\
\hline$<88.2$ & 1.000 & & 1.000 & \\
\hline$\geq 88.2$ & $0.621(0.243-1.589)$ & 0.320 & $0.895(0.533-1.501)$ & 0.895 \\
\hline
\end{tabular}

Table 2 (continued) 
Table 2 (continued)

\begin{tabular}{|c|c|c|c|c|}
\hline Variables & \multicolumn{2}{|l|}{ LRFS } & \multicolumn{2}{|l|}{ os } \\
\hline \multicolumn{5}{|c|}{$\mathrm{BED}_{10}$ of PTV min dose (Gy) } \\
\hline$<76.6$ & 1.000 & & 1.000 & \\
\hline$\geq 76.6$ & $0.216(0.063-0.743)$ & 0.015 & $1.321(0.825-2.115)$ & 0.246 \\
\hline$<83.9$ & 1.000 & & 1.000 & \\
\hline$\geq 83.9$ & $0.409(0.161-1.040)$ & 0.061 & $0.973(0.605-1.565)$ & 0.911 \\
\hline \multicolumn{5}{|c|}{ BED $_{10}$ of PTV D99 (Gy) } \\
\hline$<90.2$ & 1.000 & & 1.000 & \\
\hline$<8.8$ & 1.000 & & 1.000 & \\
\hline$\geq 8.8$ & 2.609 (1.027-6.631) & 0.044 & $1.230(0.768-1.969)$ & 0.389 \\
\hline
\end{tabular}

Hazard ratios and $95 \%$ confidence intervals were calculated by a stratified Cox proportional hazards model. P values less than 0.05 are highlighted in italic. LRFS, local recurrence-free survival; OS, overall survival; OTT, overall treatment time; CRC, colorectal cancer; PTV, planning target volume.

prognostic indicators for OS. Female $(\mathrm{P}=0.007, \mathrm{HR}=0.434)$ and tumor diameter $\geq 1.35 \mathrm{~cm}(\mathrm{P}=0.002, \mathrm{HR}=0.457)$ were protective factors for OS. The forest map was shown in Figure S2, and the nomogram used to predict the combined effect on OS at 12, 24, and 36 months was shown in Figure $S 3$.

\section{Clinical and dose parameters for LRFS}

At the time of analysis, we observed LR of 19 lesions accounting for $12.8 \%$ of the patients. Six patients were diagnosed with recurrence through biopsy, and the others were confirmed by PET-CT scan. The median LRFS was 56.9 months (95\% CI: 52.0-60.7 months). The LRFS rate was $93.6 \%, 83.5 \%, 81.4 \%$, and $76.6 \%$ at $1,2,3$, and 5 years, respectively (Figure 1).

For the 19 patients with LR after SBRT, the median follow-up time was 21.7 months (range, 4.2-67.1 months), and the median time between the beginning of SBRT and LR was 15.0 months (range, 4.2-40.0 months). The primary tumors for patients suffering local failure included 8 lesions from CRC cancer, 3 lesions from cervical cancer, 3 lesions from esophageal cancer, 2 lesion from lung cancer, 1 lesion from breast cancer, and 2 lesions from head and neck cancer
(Table S1).

Predictive factors related to LRFS are shown in Table 2. In univariate analysis, age $\geq 63$ years old, primary site of CRC cancer, prescription $\mathrm{BED}_{10}<85.2 \mathrm{~Gy}$, pathological type of adenocarcinoma, prescription PTV min $\mathrm{BED}_{10}$ $<76.6 \mathrm{~Gy}$, and GTV volume $\geq 8.8 \mathrm{~cm}^{3}$, were significantly associated with LRFS. The Kaplan-Meier analysis for local control is shown in Figure S4. In multivariate analysis (Table 3), age $\geq 63$ years $(\mathrm{P}=0.005$, $\mathrm{HR}=4.254)$, patients with primary site of $\mathrm{CRC}(\mathrm{P}=0.046, \mathrm{HR}=2.611)$, and PTV $\min \mathrm{BED}_{10}<76.6 \mathrm{~Gy}(\mathrm{P}=0.023, \mathrm{HR}=4.202)$ were poor prognostic indicators for LRFS. The forest map illustrates the risk factors and protective factors, along with and HR and 95\% CI for LRFS (Figure 2). The nomogram was established based on the results of multivariate Cox regression analysis of LRFS to predict the combined effect of influencing factors at 12, 24, and 36 months (Figure 3).

\section{Toxicities}

The most common toxicity was pneumonitis, which occurred in 17 patients $(20.2 \%)$, 5 of whom $(6.0 \%)$ experienced grade 2 pneumonitis and 12 of whom (14.2\%) experienced grade 1 pneumonitis. Another toxicity was chest 
Table 3 Multivariable Cox analysis of LRFS and OS

\begin{tabular}{|c|c|c|c|c|}
\hline Variables & \multicolumn{2}{|l|}{ LRFS } & \multicolumn{2}{|l|}{ os } \\
\hline \multicolumn{5}{|l|}{ Age (years) } \\
\hline$<63$ & 1.000 & & 1.000 & \\
\hline$\geq 63$ & $4.254(1.563-11.574)$ & 0.005 & 1.793 (1.097-2.929) & 0.020 \\
\hline Non-CRC & 1.000 & & & \\
\hline CRC & $2.611(1.019-6.693)$ & 0.046 & & \\
\hline $\mathrm{BED}_{10}$ of PTV min dose (Gy) & & & - & - \\
\hline$<76.6$ & 1.000 & & & \\
\hline Male & & & 1.000 & \\
\hline Female & & & $0.434(0.236-0.796)$ & 0.007 \\
\hline Tumor diameter (cm) & - & - & & \\
\hline$<1.35$ & & & 1.000 & \\
\hline$\geq 1.35$ & & & $0.457(0.275-0.758)$ & 0.002 \\
\hline Histology & - & - & & \\
\hline Others & & & 1.000 & \\
\hline Adenocarcinoma & & & $2.354(1.122-4.942)$ & 0.024 \\
\hline
\end{tabular}

Hazard ratios and $95 \%$ confidence intervals were calculated by a stratified Cox proportional hazards model. P values less than 0.05 are highlighted in italic. LRFS, Local recurrence-free survival; OS, overall survival; CRC, colorectal cancer.

pain, which occurred in 2 patients $(2.4 \%)$, with 1 patient (1.2\%) experiencing grade 2 chest pain and 1 (1.2\%) experiencing grade 1 chest pain. No patient developed acute grade 3 and 4 toxicity, and there were no treatment-related deaths (Table S2).

\section{Discussion}

This single-institution retrospective study analyzed the clinical factors and dose parameters affecting local control in patients with a controlled primary tumor and up to five lung metastatic lesions treated by SBRT. The results confirm that SBRT is associated with outstanding local control and consistent with reported local control rates using SBRT for lung oligometastases (range of 70-90\% at $2-3$ years) $(11,22-24)$. For the first time, we report the optimal dose parameters for pulmonary oligometastases treated by SBRT from a radiation oncology perspective.

SBRT is a modern radiation therapy technique characterized by delivering a highly conformal ablative dose in a few fractions while sparing the surrounding healthy tissues. Currently, there is no consensus on the optimal SBRT dose for pulmonary oligometastases. Ricco et al. found that an SBRT doses of $\mathrm{BED}_{10} \geq 100$ Gy had a 3-year LC rate of $77.1 \%$ compared to $45.0 \%$ for lung metastases treated with $\mathrm{BED}_{10}<100$ Gy $(\mathrm{P}<0.01)(17)$. Another study indicated that $\mathrm{BED}_{10}>72$ Gy yielded better local control (90.0\% vs. $57 \%$ at 1 year) (18). A German study showed that BEDiso at PTV isocenter $\geq 130$ Gy showed a trend for superior LC $(\mathrm{P}=0.054)$ (25). However, it is particularly challenging to compare clinical outcomes only by prescription dose when different dose regimens 


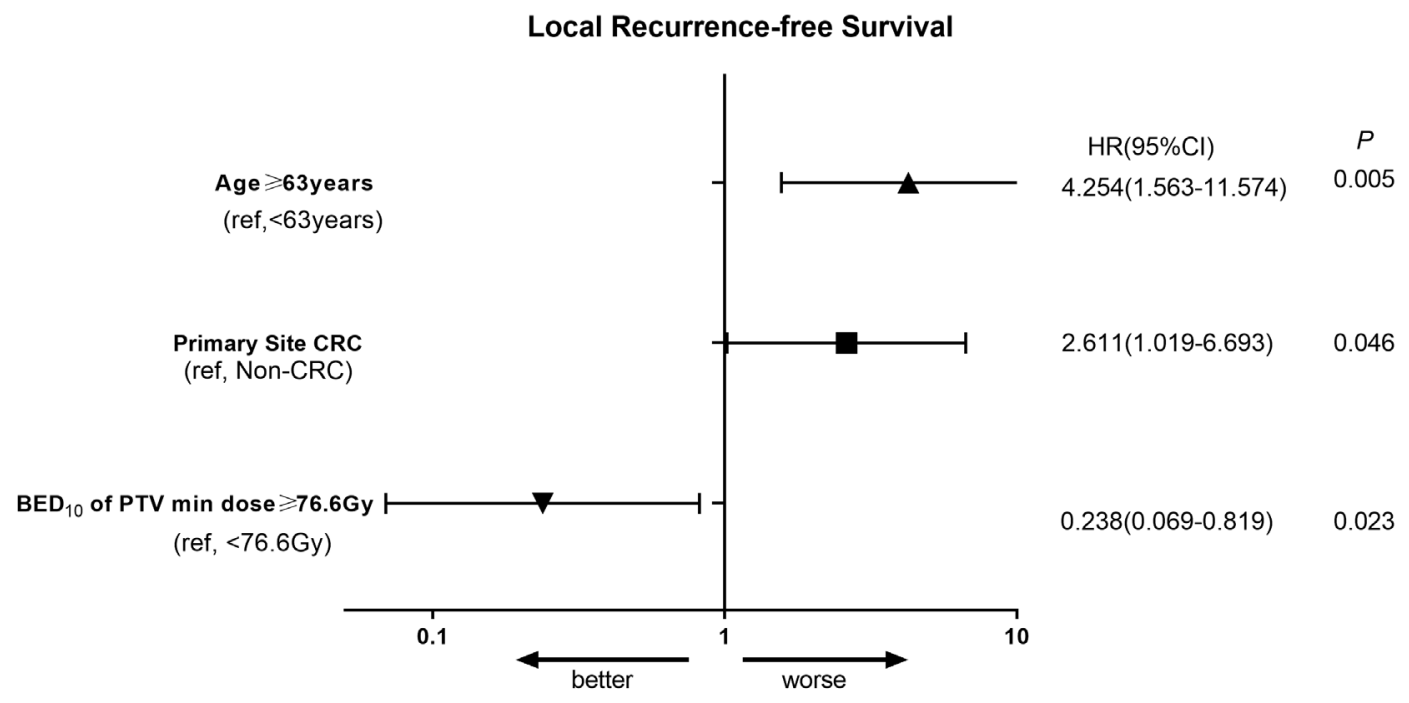

Figure 2 Forest map of the risk and protective factors for local recurrence-free survival (LRFS), along with their hazard ratios (HRs) and 95\% confidence interval (CI). Univariate and multivariate analysis with the Cox proportional hazards model was used to investigate the effect of different factors on LRFS. Covariates in multivariate analysis included age ( $\geq 63 v s .<63$ years), primary site (CRC vs. non-CRC), and PTV min $\mathrm{BED}_{10}(\geq 76.6$ vs. <76.6 Gy).

Points
Age
Primary Site
BED $_{10}$ of PTV min dose
Total Points
Linear Predictor
1-year LRFS Probability
2-year LRFS Probability
3-year LRFS Probability

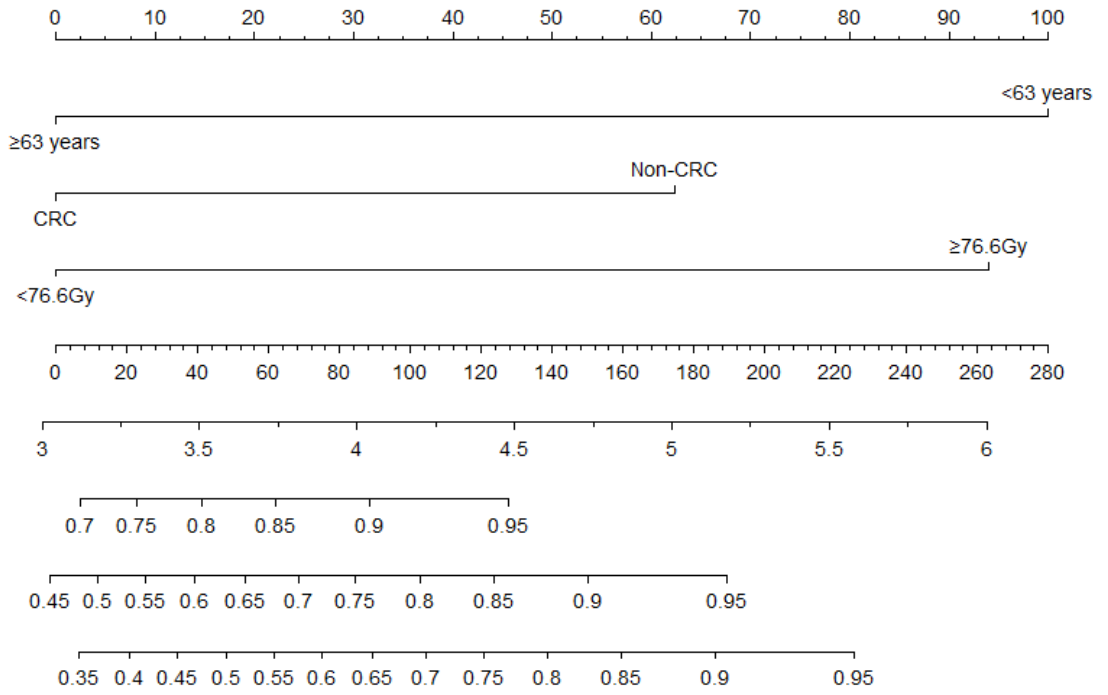

Figure 3 Nomogram based on the results of multivariate Cox regression analysis of local recurrence-free survival (LRFS). The 1-, 2- and 3 -year of LRFS probability in patients was estimated with the prognostic factors. Draw an upward vertical line from the covariate to the points bar to calculate points, based on the sum of the covariate points, than draw a downward vertical line from the total points line to calculate LRFS.

are used. Different prescription methods were used in each SBRT study, and our previous paper found that even if the prescription dose is the same, the dose delivered to PTV could vary according to different prescription methods and isodose line selection. Therefore, we proposed both PTVD95 $\mathrm{BED}_{10}>86$ Gy and PTVmean $\mathrm{BED}_{10}>130$ Gy for SBRT plan optimization (19).

This study is unique when compared to other recently 
published studies, and, for the first time, reports detailed optimal dose parameters for pulmonary oligometastases treated by SBRT (prescription $\mathrm{BED}_{10}, \mathrm{BED}_{10}$ of PTV max dose, $\mathrm{BED}_{10}$ of PTV mean dose, mean PTV dose, $\mathrm{BED}_{10}$ of PTV min dose, $\mathrm{BED}_{10}$ of PTV D95, and $\mathrm{BED}_{10}$ of PTV D99) from a radiation oncology perspective. We found that prescription $\mathrm{BED}_{10}<85.2 \mathrm{~Gy}$ and prescription PTV min $\mathrm{BED}_{10}<76.6$ Gy were significantly associated with LR. In multivariate analysis, we found that PTV min $\mathrm{BED}_{10}<76.6 \mathrm{~Gy}$ was a poor prognostic indicator for LRFS. Our findings suggest that multiple dose parameters, instead of prescription dose only, should be considered for optimal local control of pulmonary oligometastases treated by SBRT. A modeling study also showed that BEDave (the average between near-minimum and nearmaximum doses) was better correlated with tumor control than either BEDmax or BEDmin (26). Therefore, more robust dose prediction models are needed for pulmonary oligometastases treated by SBRT.

Many studies have reported that LRFS was different between pulmonary oligometastases from CRC and primary NSCLCs. A matched-pair analysis compared the outcome of SBRT of pulmonary oligometastases from CRC and early stage NSCLCs. It revealed that LRFS for pulmonary oligometastases from CRC was significantly worse than that of NSCLC when treated with 48-60 Gy/4-5 frequency. The 1-, 3-, and 5-year LRFS of oligometastatic lung tumors from CRC vs. NSCLC were $80.6 \%$ vs. $100.0 \%, 68.6 \%$ vs. $97.2 \%$, and $68.6 \%$ vs. $81.0 \%$, respectively (27). A Japanese study showed that the 3-year LRFS rate of patients with CRC was significantly worse $(39.0 \%)$ than that of noncolorectal cancers $(82.0 \%)$, and the CRC origin was the only significant prognostic factor for local control (28). Takeda $e t a l$. also reported the 2-year LC rate of pulmonary oligometastases from CRC was worse than that of noncolorectal histology (80.0\% vs. 94.0\%) (29). In our study, the SBRT dose regimen was well balanced between the primary colorectal cancer and non-colorectal cancer group (data not shown), while the LRFS of patients with colorectal cancer metastasis at 1,2 , and 3 years was significantly worse than those with a non-CRC histology $(81.0 \%, 59.0 \%$, and $59.0 \%$, vs. $96.4 \%, 88.8 \%$, and $86.2 \%$, respectively) $(\mathrm{P}=0.003)$. Multivariate analysis showed that patients with CRC histology was a poor prognostic indicator for LRFS ( $\mathrm{P}=0.046, \mathrm{HR}=2.611)$. A higher prescription dose was correlated with better local control with borderline significance in the non-CRC group $(\mathrm{P}=0.055)$ as compared to the CRC group $(\mathrm{P}>0.05)$. This study suggests that $\mathrm{CRC}$ - derived pulmonary oligometastases are radioresistant, while the optimal dose remains unclear. Another study reported no significant difference in LC observed when the prescription $\mathrm{BED}_{10}$ was over $100 \mathrm{~Gy}$ for CRC versus non-CRC histology (30). However, our study found that there was no significant difference of OS between primary CRC patients and non-CRC patients (1-, 2-, and 3-year OS was $66.7 \%, 66.7 \%, 66.7 \%$, and $76.5 \%, 58.2 \%$, and $46.7 \%$, respectively; $\mathrm{P}=0.969)$. Another study also indicated that CRC patients had better OS as compared to patients of other histologies (31). The favorable OS for pulmonary oligometastases with primary colorectal histology can be partially explained by the effective systemic treatment agents and aggressive local therapy.

Due to its retrospective nature, this study has some limitations, including the potential confounding factors and limited number of patients that might have influenced the final conclusion of the study. We do not include centrally located pulmonary oligometastasis due to its relatively low prescription dose, and further study is needed to validate the role of SBRT in this setting when normal tissues are carefully protected. Furthermore, multiple primary histologies, different dose-fraction regimens, and a variety of systemic agents were used in this study. Therefore, a well-designed prospective randomized control trial or matched-pair analysis is warranted to determine the optimal SBRT regimen for predictable survival benefits in select patients.

\section{Conclusions}

Lung SBRT is a feasible treatment option for patients with pulmonary oligometastasis derived from multiple primary histologies, with high local control rates and minimal toxicity. Multiple dose parameters, rather than prescription dose alone, should be considered for optimal local control of pulmonary oligometastases treated by SBRT.

\section{Acknowledgments}

Funding: This work was supported by the National Natural Science Foundation of China Grants (No. 81872699) and the Key Project of Shaanxi Province (No. 2017ZDXMSF-043).

\section{Footnote}

Reporting Checklist: The authors have completed the 
STROBE reporting checklist. Available at http://dx.doi. org/10.21037/tlcr-20-867

Data Sharing Statement: Available at http://dx.doi. org/10.21037/tlcr-20-867

Conflicts of Interest: All authors have completed the ICMJE uniform disclosure form (available at http://dx.doi. org/10.21037/tlcr-20-867). The authors have no conflicts of interest to declare.

Ethical Statement: The authors are accountable for all aspects of the work in ensuring that questions related to the accuracy or integrity of any part of the work are appropriately investigated and resolved. The analysis is in compliance with the principles of the Declaration of Helsinki (as revised in 2013). This study was approved by the institutional review board of Xijing Hospital (no. KY20202002-F-1). Written informed consent was obtained from each of the participants.

Open Access Statement: This is an Open Access article distributed in accordance with the Creative Commons Attribution-NonCommercial-NoDerivs 4.0 International License (CC BY-NC-ND 4.0), which permits the noncommercial replication and distribution of the article with the strict proviso that no changes or edits are made and the original work is properly cited (including links to both the formal publication through the relevant DOI and the license). See: https://creativecommons.org/licenses/by-nc-nd/4.0/.

\section{References}

1. Hellman S, Weichselbaum RR. Oligometastases. J Clin Oncol 1995;13:8-10.

2. Gomez DR, Blumenschein GR Jr, Lee JJ, et al. Local consolidative therapy versus maintenance therapy or observation for patients with oligometastatic non-smallcell lung cancer without progression after first-line systemic therapy: a multicentre, randomised, controlled, phase 2 study. Lancet Oncol 2016;17:1672-82.

3. Gomez DR, Tang C, Zhang J, et al. Local Consolidative Therapy Vs. Maintenance Therapy or Observation for Patients With Oligometastatic Non-Small-Cell Lung Cancer: Long-Term Results of a Multi-Institutional, Phase II, Randomized Study. J Clin Oncol 2019;37:1558-65.

4. Timmerman RD, Herman J, Cho LC. Emergence of stereotactic body radiation therapy and its impact on current and future clinical practice. J Clin Oncol 2014;32:2847-54.

5. Timmerman RD, Paulus R, Pass HI, et al. Stereotactic Body Radiation Therapy for Operable Early-Stage Lung Cancer: Findings From the NRG Oncology RTOG 0618 Trial. JAMA Oncol 2018;4:1263-6.

6. Thompson M, Rosenzweig KE. The evolving toxicity profile of SBRT for lung cancer. Transl Lung Cancer Res 2019;8:48-57.

7. Chang JY, Senan S, Paul MA, et al. Stereotactic ablative radiotherapy versus lobectomy for operable stage I nonsmall-cell lung cancer: a pooled analysis of two randomised trials. Lancet Oncol 2015;16:630-7.

8. Palma DA, Olson R, Harrow S, et al. Stereotactic ablative radiotherapy versus standard of care palliative treatment in patients with oligometastatic cancers (SABRCOMET): a randomised, phase 2, open-label trial. Lancet 2019;393:2051-8.

9. Rusthoven KE, Kavanagh BD, Burri SH, et al. Multiinstitutional phase I/II trial of stereotactic body radiation therapy for lung metastases. J Clin Oncol 2009;27:1579-84.

10. Tanadini-Lang S, Rieber J, Filippi AR, et al. Nomogram based overall survival prediction in stereotactic body radiotherapy for oligo-metastatic lung disease. Radiother Oncol 2017;123:182-8.

11. Navarria P, Ascolese AM, Tomatis S, et al. Stereotactic body radiotherapy (SBRT) in lung oligometastatic patients: role of local treatments. Radiat Oncol 2014;9:91.

12. Nuyttens JJ, van der Voort van Zyp NC, Verhoef C, et al. Stereotactic body radiation therapy for oligometastases to the lung: a phase 2 study. Int J Radiat Oncol Biol Phys 2015;91:337-43.

13. Franceschini D, De Rose F, Franzese C, et al. Predictive Factors for Response and Survival in a Cohort of Oligometastatic Patients Treated With Stereotactic Body Radiation Therapy. Int J Radiat Oncol Biol Phys 2019;104:111-21.

14. Guckenberger M, Wulf J, Mueller G, et al. Doseresponse relationship for image-guided stereotactic body radiotherapy of pulmonary tumors: relevance of $4 \mathrm{D}$ dose calculation. Int J Radiat Oncol Biol Phys 2009;74:47-54.

15. Nagata $Y$, Hiraoka M, Shibata T, et al. Prospective Trial of Stereotactic Body Radiation Therapy for Both Operable and Inoperable T1N0M0 Non-Small Cell Lung Cancer: Japan Clinical Oncology Group Study JCOG0403. Int J Radiat Oncol Biol Phys 2015;93:989-96.

16. Pareek V. Assessment of toxicity and clinical outcomes with SBRT in lung metastases: A single institute experience. 
Ann Oncol 2019;30 Suppl 2:ii69.

17. Ricco A, Davis J, Rate W, et al. Lung metastases treated with stereotactic body radiotherapy: the RSSearch ${ }^{\circledR}$ patient Registry's experience. Radiat Oncol 2017;12:35.

18. Wegner RE, Abel S, Hasan S, et al. Stereotactic Body Radiotherapy (SBRT) for Oligometastatic Lung Nodules: A Single Institution Series. Front Oncol 2019;9:334.

19. Zhao L, Zhou S, Balter P, et al. Planning Target Volume D95 and Mean Dose Should Be Considered for Optimal Local Control for Stereotactic Ablative Radiation Therapy. Int J Radiat Oncol Biol Phys 2016;95:1226-35.

20. Yamamoto T, Niibe Y, Yamada K, et al. Significant reduction of oncologic pulmonary death by local control for pulmonary oligometastases treated with stereotactic body radiotherapy. Radiother Oncol 2020;147:86-91.

21. Zhang X, Liu H, Balter P, et al. Positron emission tomography for assessing local failure after stereotactic body radiotherapy for non-small-cell lung cancer. Int J Radiat Oncol Biol Phys 2012;83:1558-65.

22. García-Cabezas S, Bueno C, Rivin E, R et al. Lung metastases in oligometastatic patients: outcome with stereotactic body radiation therapy (SBRT). Clin Transl Oncol 2015;17:668-72.

23. Song A, Lu B. Utility of stereotactic ablative radiotherapy/ stereotactic body radiation therapy in the setting of oligometastatic non-small cell lung cancer. J Thorac Dis 2018;10:657-60.

24. Baschnagel AM, Mangona VS, Robertson JM, et al. Lung metastases treated with image-guided stereotactic body radiation therapy. Clin Oncol (R Coll Radiol) 2013;25:236-41.

25. Hoerner-Rieber J, Duma M, Blanck O, et al. Stereotactic body radiotherapy (SBRT) for pulmonary metastases from renal cell carcinoma-a multicenter analysis of the German working group "Stereotactic Radiotherapy". J Thorac Dis 2017;9:4512-22.

26. Klement RJ, Sonke JJ, Allgäuer M, et al. Correlating Dose Variables with Local Tumor Control in Stereotactic Body Radiation Therapy for Early- Stage Non-Small Cell Lung Cancer: A Modeling Study on 1500 Individual Treatments. Int J Radiat Oncol Biol Phys 2020;107:579-86.

27. Wang X, Zamdborg L, Ye H, et al. A matched-pair analysis of stereotactic body radiotherapy (SBRT) for oligometastatic lung tumors from colorectal cancer versus early stage non-small cell lung cancer. BMC Cancer 2018;18:962.

28. Lo SS, Teh BS, Mayr NA, et al. Stereotactic body radiation therapy for oligometastases. Discov Med 2010;10:247-54.

29. Takeda A, Kunieda E, Ohashi T, et al. Stereotactic body radiotherapy (SBRT) for oligometastatic lung tumors from colorectal cancer and other primary cancers in comparison with primary lung cancer. Radiother Oncol 2011;101:255-9.

30. Sharma A, Duijm M, Oomen-de Hoop E, et al. Factors affecting local control of pulmonary oligometastases treated with stereotactic body radiotherapy. Acta Oncol 2018;57:1031-7.

31. Sharma A, Duijm M, Oomen-de Hoop E, et al. Survival and prognostic factors of pulmonary oligometastases treated with stereotactic body radiotherapy. Acta Oncol 2019;58:74-80.

(English Language Editor: J. Gray)
Cite this article as: Chai G, Yin Y, Zhou X, Hu Q, Lv B, Li Z, Shi M, Zhao L. Pulmonary oligometastases treated by stereotactic body radiation therapy (SBRT): a single institution's experience. Transl Lung Cancer Res 2020;9(4):1496-1506. doi: 10.21037/tlcr-20-867 


\section{Supplementary}

A

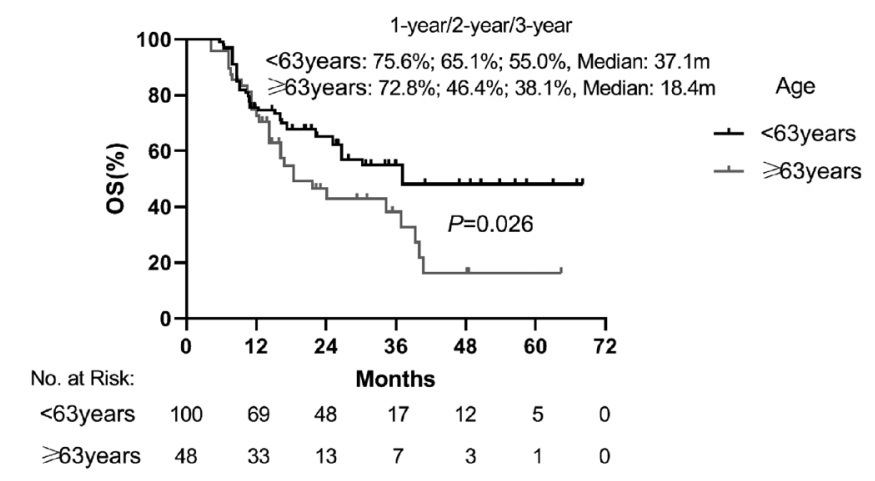

C

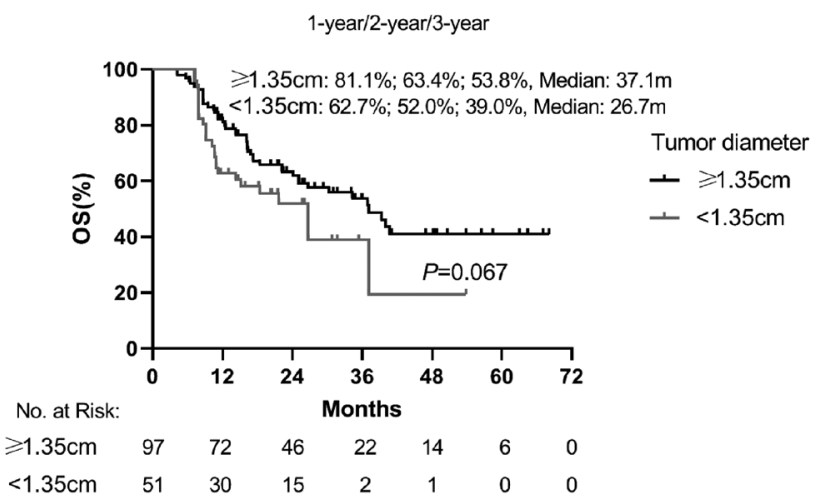

B

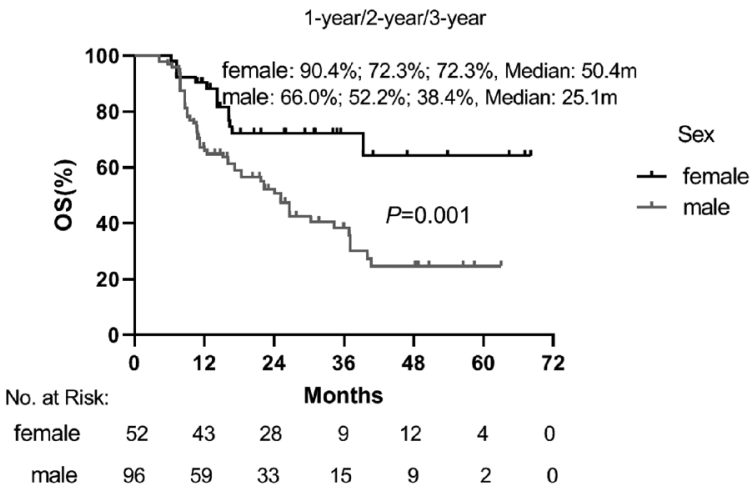

D

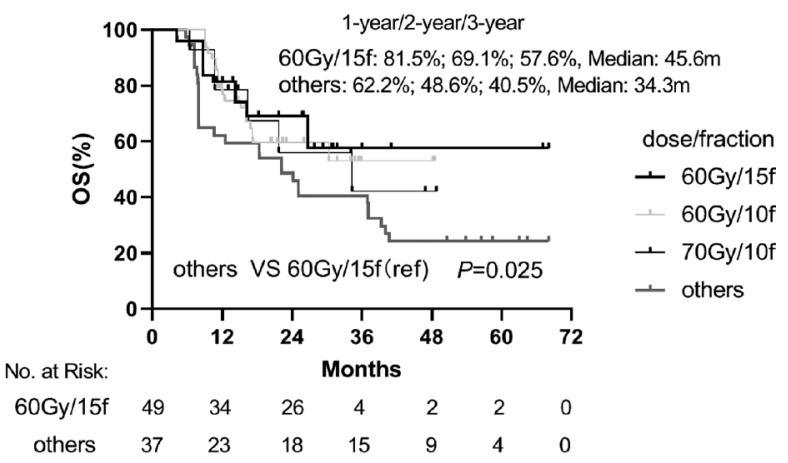

E

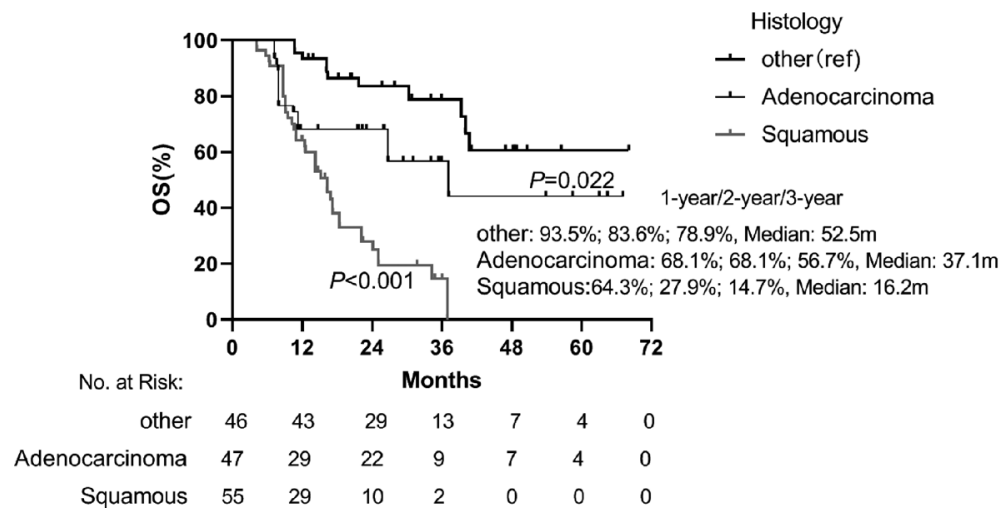

Figure S1 Kaplan-Meier analysis of overall survival (OS) in different subgroups, age (A); sex (B); tumor diameter (C); dose/fraction (D); histology (E). The median OS time in different subgroups was showed, the percent survival of 1-, 2- and 3-year of OS rate and the number at risk was also presented, respectively. 


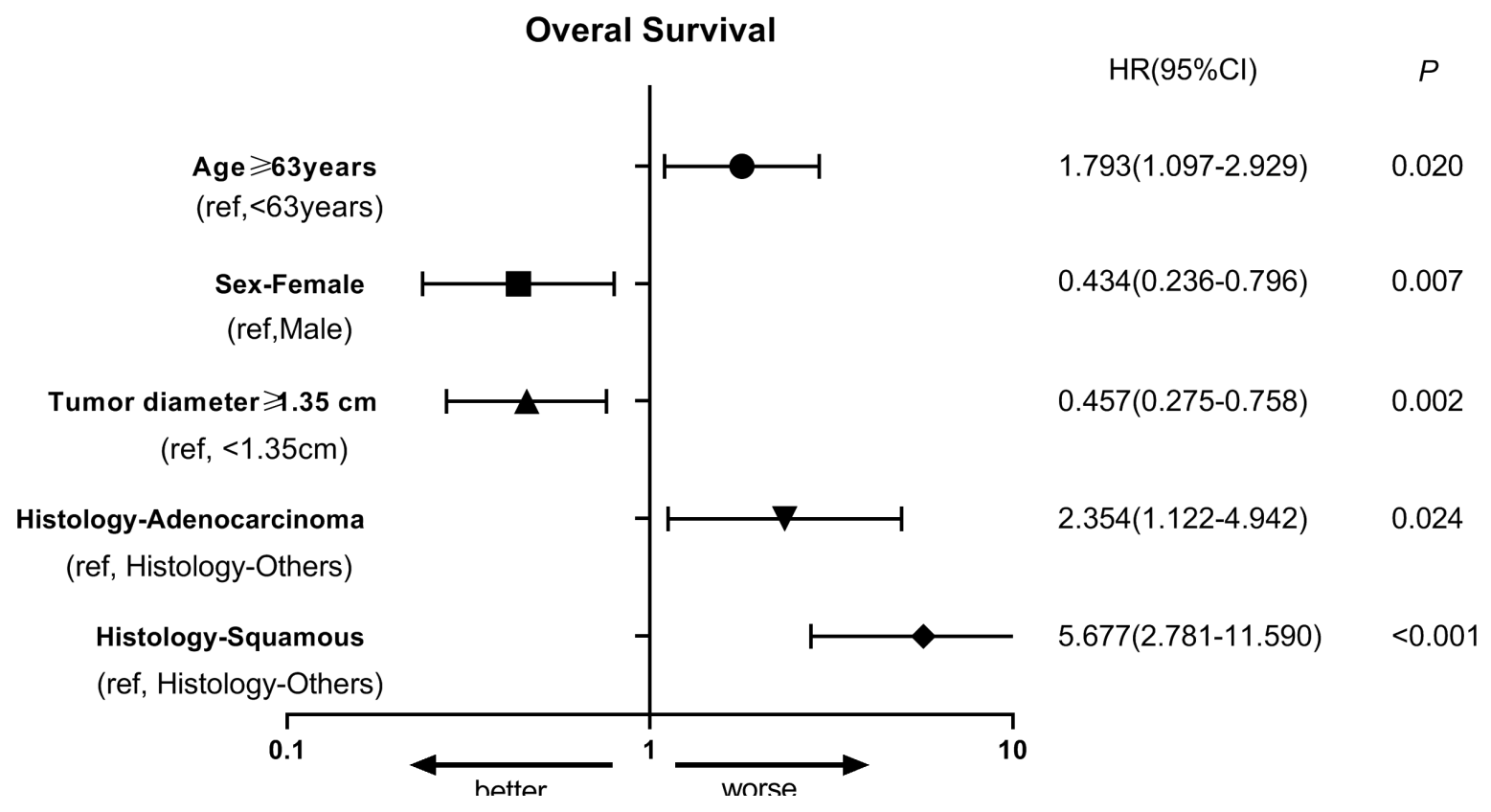

Figure S2 Forest map of the risk and protective factors for overall survival (OS), along with their hazard ratios (HRs) and 95\% confidence interval (CI). Univariate and multivariate analysis with the Cox proportional hazards model was used to investigate the effect of different factors on OS. Covariates in multivariate analysis included age ( $\geq 63 v s .<63$ years), sex (female $v s$. male), tumor diameter $(\geq 1.35 v s .<1.35 \mathrm{~cm})$, and histology (adenocarcinoma vs. squamous vs. others).

Points

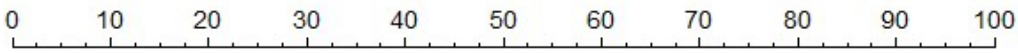

Age
Sex
Tumor diameter
Histology
Total Points
Linear Predictor
1-year OS Probability
2-year OS Probability
3-year OS Probability
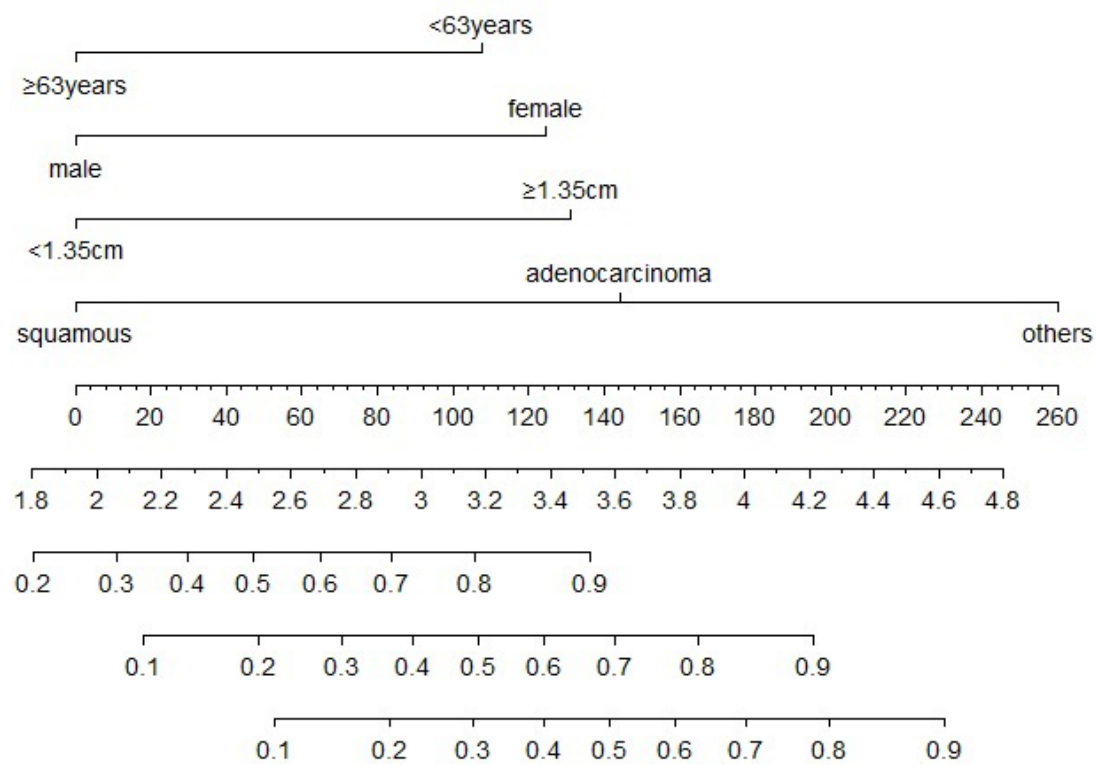

Figure S3 Nomogram based on the results of multivariate Cox regression analysis of overall survival (OS). The 1-, 2- and 3-year of OS probability in patients was estimated with the prognostic factors. Draw an upward vertical line from the covariate to the points bar to calculate points, based on the sum of the covariate points, than draw a downward vertical line from the total points line to calculate OS. 
A

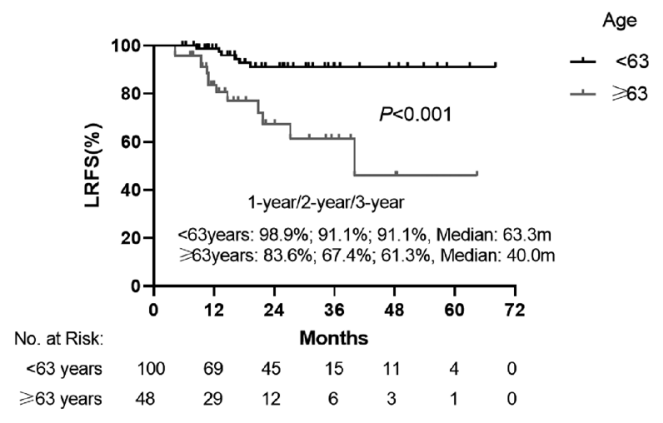

C

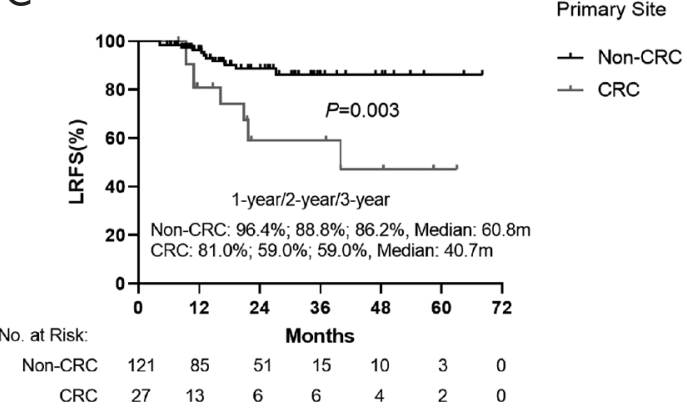

$E$

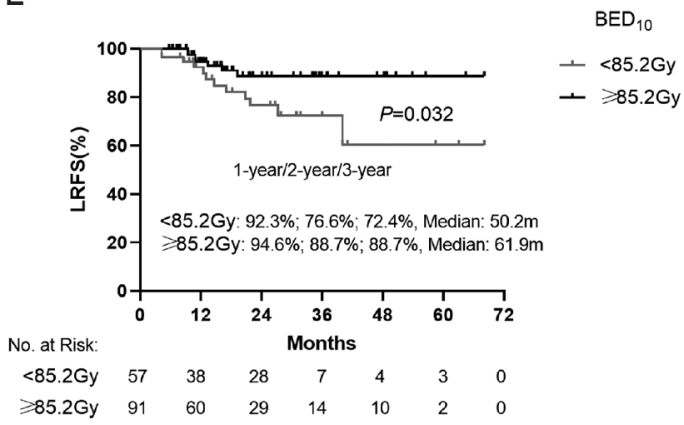

B
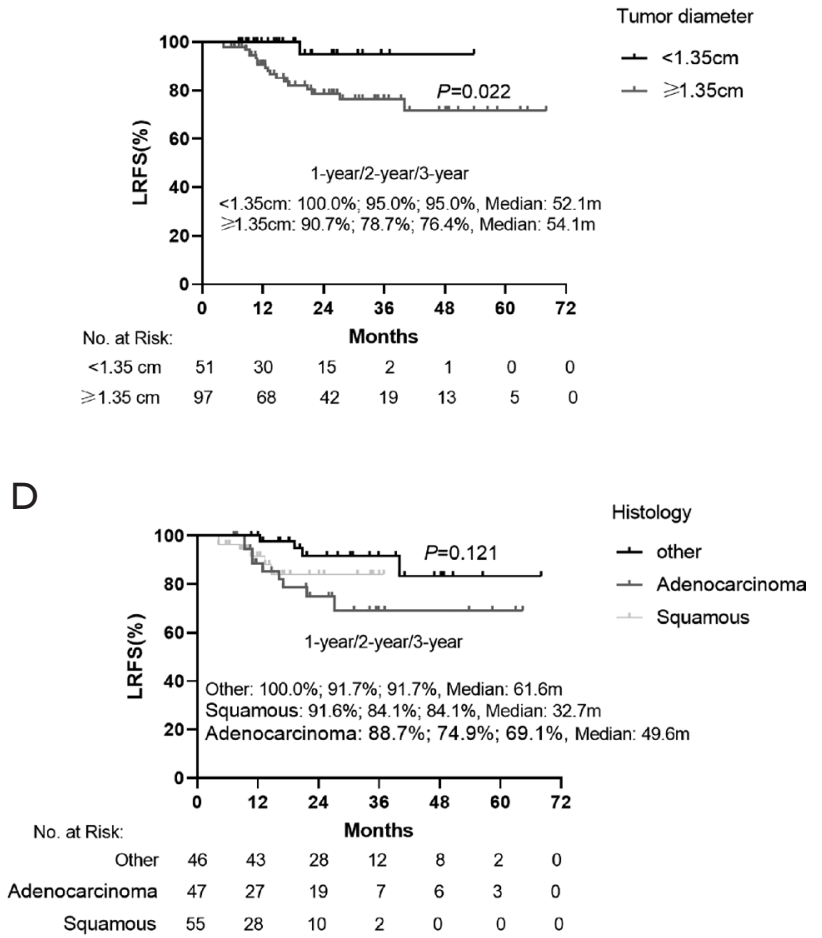

$\mathrm{F}$

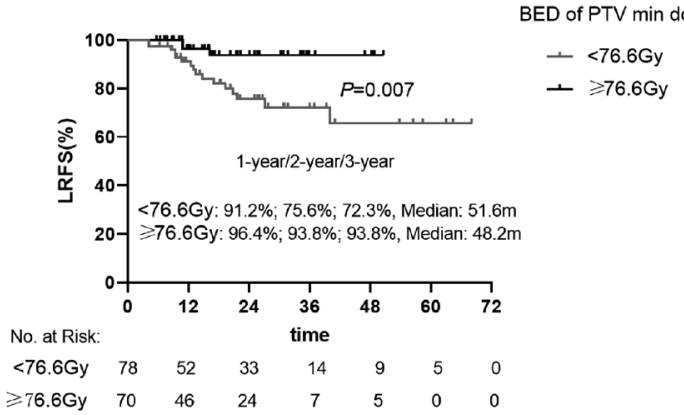

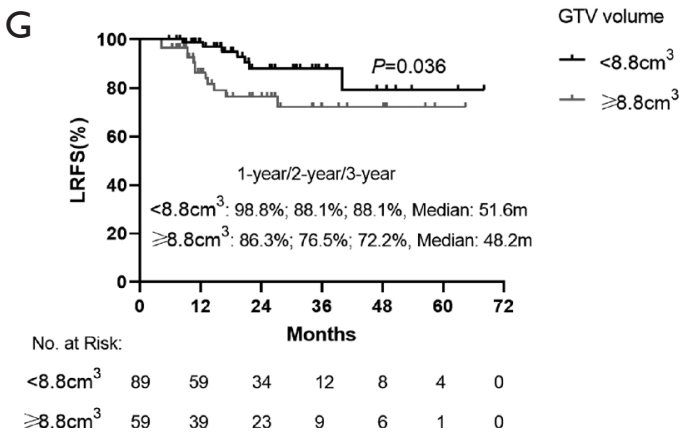

Figure S4 Kaplan-Meier analysis of local recurrence-free survival (LRFS) in different subgroups, age (A); tumor diameter (B); primary site (C); histology (D); $\mathrm{BED}_{10}(\mathrm{E}) ; \mathrm{BED}_{10}$ of PTV min dose (F); GTV volume (G). The median LRFS time in different subgroups was showed, the percent survival of 1-, 2- and 3-year of LRFS rate and the number at risk was also presented, respectively. 
Table S1 The failure patterns after SBRT

\begin{tabular}{lc}
\hline Failure patterns & No. (\%) \\
\hline Number of patients & 84 \\
Death & $37(44.1)$ \\
Local failure & $19(12.8)$ \\
Colorectal primary & $8(5.4)$ \\
Cervical primary & $3(2.0)$ \\
Esophagus primary & $3(2.0)$ \\
Lung primary & $2(1.4)$ \\
Breast primary & $1(0.7)$ \\
Head and neck primary & $2(1.4)$ \\
Intrathoracic distant failure & $9(10.7)$ \\
Extrathoracic distant failure & $37(44.1)$ \\
\hline The data was shown as number (percent). SBRT, stereotactic \\
body radiation therapy.
\end{tabular}

Table S2 Toxicity after stereotactic body radiation therapy for pulmonary metastases

\begin{tabular}{lccc}
\hline Toxic effects $(\mathrm{n}=84)$ & $\begin{array}{c}\text { Total, } \\
\mathrm{n}(\%)\end{array}$ & $\begin{array}{c}\text { Grade 1, } \\
\mathrm{n}(\%)\end{array}$ & $\begin{array}{c}\text { Grade 2, } \\
\mathrm{n}(\%)\end{array}$ \\
\hline Hemoptysis & 0 & 0 & 0 \\
Rib fracture & 0 & 0 & 0 \\
Brachial plexus & 0 & 0 & 0 \\
Chest pain & $2(2.4)$ & $1(1.2)$ & $1(1.2)$ \\
Pulmonary & $17(20.2)$ & $12(14.2)$ & $5(6.0)$ \\
\hline
\end{tabular}

The data was shown as number (percent). Toxicity was graded according to the Common Terminology Criteria for Adverse Events (CTCAE) v4.0. 